\title{
LOCAL SPECTRAL PROPERTY OF RELATIVELY REGULAR OPERATORS
}

\author{
Eungil Ko And MeE-Jung LeE
}

Abstract. In this paper, we study some relatively regular operators $T$ such that $T=T S T$ for some $S \in \mathscr{L}(\mathscr{H})$. We give some spectral and local spectral properties between $T$ and $S$. We also show that some relatively regular operators $T$ have a nontrivial invariant subspace. Finally, we introduce and study the local spectral property of relatively regular operators modulo a nilpotent operator.

Mathematics subject classification (2010): 47B20, 47A10.

Keywords and phrases: Relatively regular operator, local spectral property.

\section{REFERENCES}

[1] P. AiEnA, Fredholm and local spectral theory with applications to multipliers, Kluwer Acad. Pub., 2004.

[2] F. V. AtKinson, On relatively regular operators, Acta Sci. Math. (Szeged) 15 (1953), 38-56.

[3] S. R. Caradus, Mapping properties of relatively regular operators, Proc. Amer. Math. Soc. 47 (1975), 409-412.

[4] I. ColojoARA AND C. FoiAs, Theory of generalized spectral operators, Gordon and Breach, New York, 1968.

[5] S. Djordjević And Y. M. Han, A note on Weyl's theorem for operator matrices, Proc. Amer. Math. Soc. 131 (2003), 2543-2547.

[6] I. KAPLANSKY, Regular Banach algebras, J. Indian Math. Soc. 12 (1948), 57-62.

[7] Y. Kim, E. Ko, AND J. LEE, Operators with the single-valued extension property, Bull. Kor. Math. Soc. 43 (2006), no. 3, 509-517.

[8] E. Ko, Algebraic and triangular n-hyponormal operators, Proc. Amer. Math. Soc. 123 (1995), $3473-$ 3481.

[9] R. LANGe AND S. WANG, New approaches in spectral decomposition, Contemporary Math. 128, Amer. Math. Soc., 1992.

[10] K. B. Laursen And M. M. Neumann, An introduction to Local spectral theory, London Math. Soc. Monograghs New Series, 20, Claredon Press, Oxford, 2000.

[11] C. Schmoeger, Charaterizations of some classes of relatively regular operators, Linear Algebra Appl. 429 (2008), 302-310. 